ACCEPTED By THE ASTROPHYSICAL JOURNAL

Preprint typeset using $\mathrm{LT}_{\mathrm{E}} \mathrm{X}$ style emulateapj v. 7/15/03

\title{
DETECTION OF LOW-HARD STATE SPECTRAL AND TIMING SIGNATURES FROM THE BLACK HOLE X-RAY TRANSIENT XTE J1650-500 AT LOW X-RAY LUMINOSITIES
}

\author{
John A. TOMSICK ${ }^{1}$, EmRah KALEMCI ${ }^{2}$, Philip KAARET ${ }^{3}$ \\ Accepted by the Astrophysical Journal
}

\begin{abstract}
Using the Chandra X-ray Observatory and the Rossi X-ray Timing Explorer, we have studied the black hole candidate (BHC) X-ray transient XTE J1650-500 near the end of its 2001-2002 outburst after its transition to the low-hard state at X-ray luminosities down to $L=1.5 \times 10^{34} \mathrm{erg} \mathrm{s}^{-1}(1-9 \mathrm{keV}$, assuming a source distance of $4 \mathrm{kpc}$ ). Our results include a characterization of the spectral and timing properties. At the lowest sampled luminosity, we used an $18 \mathrm{ks}$ Chandra observation to measure the power spectrum at low frequencies. For the 3 epochs at which we obtained Chandra/RXTE observations, the $0.5-20 \mathrm{keV}$ energy spectrum is consistent with a spectral model consisting of a power-law with interstellar absorption. We detect evolution in the power-law photon index from $\Gamma=1.66 \pm 0.05$ to $\Gamma=1.93 \pm 0.13$ (90\% confidence errors), indicating that the source softens at low luminosities. The power spectra are characterized by strong (20-35\% fractional rms) band-limited noise, which we model as a zero-centered Lorentzian. Including results from an RXTE study of XTE J1650-500 near the transition to the low-hard state by Kalemci et al. (2003), the half-width of the zero-centered Lorentzian (roughly where the band-limited noise cuts off) drops from $4 \mathrm{~Hz}$ at $L=7 \times 10^{36} \mathrm{erg} \mathrm{s}^{-1}$ (1-9 keV, absorbed) to $0.067 \pm 0.007 \mathrm{~Hz}$ at $L=9 \times 10^{34} \mathrm{erg} \mathrm{s}^{-1}$ to $0.0035 \pm 0.0010 \mathrm{~Hz}$ at the lowest luminosity. While the spectral and timing parameters evolve with luminosity, it is notable that the general shapes of the energy and power spectra remain the same, indicating that the source stays in the low-hard state. This implies that the X-ray emitting region of the system likely keeps the same overall structure, while the luminosity changes by a factor of 470 . We discuss how these results may constrain theoretical black hole accretion models.
\end{abstract}

Subject headings: accretion, accretion disks — black hole physics: general — stars: individual (XTE J1650_ 500) — stars: black holes — X-rays: stars

\section{INTRODUCTION}

To fully understand the behavior of black hole candidate (BHC) X-ray transients, it is important to determine the source properties for the full range of observed X-ray luminosities from outburst peak at $L \sim 10^{38-39} \mathrm{erg} \mathrm{s}^{-1}$ to quiescence at $L \sim 10^{30-33} \mathrm{erg} \mathrm{s}^{-1}$. The brighter part of this range has been well-studied by several satellites, and, due to its broad bandpass (2-200 keV) and its excellent timing capabilities, the Rossi X-ray Timing Explorer (RXTE, Bradt, Rothschild \& Swank 1993) has proved to be an extremely useful tool for studying the luminosity range above $\sim 10^{35-36} \mathrm{erg} \mathrm{s}^{-1}$ (for sources with typical distances of 3-10 kpc). For most systems, this is below the luminosity where the source makes a transition from the canonical high-soft state to the low-hard (or hard) state (McClintock \& Remillard 2003), and our group as well as others have used RXTE to study BHC transients as they undergo soft-to-hard state transitions during outburst decay (Tomsick \& Kaaret 2000; Nowak, Wilms \& Dove 2002; Kalemci 2002; Kalemci et al. 2003a). However, as RXTE is not an imaging instrument, background and source confusion become significant at lower luminosities, making both spectral and timing studies problematic. Thus, at low luminosities, imaging observatories such as BeppoSAX, the Chandra X-ray Observatory (Weisskopf et al. 2002), and XMM-Newton are necessary, and these satellites have been used to study BHC transients in quiescence (Garcia et al. 2001). The aim of this

\footnotetext{
${ }^{1}$ Center for Astrophysics and Space Sciences, Code 0424, University of California at San Diego, La Jolla, CA, 92093, USA (e-mail: jtomsick@ucsd.edu)

${ }^{2}$ Space Sciences Laboratory, University of California, Berkeley, CA, 94720-7450, USA

${ }^{3}$ Harvard-Smithsonian Center for Astrophysics, 60 Garden Street, Cambridge, MA, 02138, USA
}

study is to use Chandra to study the evolution of XTE J1650500 in the luminosity range between the transition to the hard state and quiescence.

Few previous studies have been dedicated to observing BHC transients at luminosities between the hard state transition and quiescence even though the source evolution has not been well-characterized, and the physical changes that occur in this regime are unclear. The results of the studies of BHC transients that have been undertaken in this regime are intriguing. A correlation between X-ray and radio flux has been found for several BHC systems in the hard state (Corbel et al. 2003; Gallo. Fender \& Poolev 2003), and this has been interpreted as evidence for a connection between $\mathrm{X}$ ray production and a compact radio jet (Markoff et al. 2003). As the sources decay, it has been observed that the X-ray energy spectra maintain a power-law shape; however, in some cases, there is evidence for spectral evolution with sources becoming softer at low luminosities (Ebisawa et al. 1994; Tomsick. Corbel \& Kaaret 2001; Corbel et al. 2003; Kalemci 2002). X-ray timing studies immediately after the hard state transition show that, in most cases, the characteristic frequencies drop (Tomsick \& Kaaret 2000; Nowak. Wilms \& Dove 2002; Kalemci et al. 2003a), and this may indicate movement of the inner edge of the optically thick accretion disk away from the black hole. However, for the most part, the X-ray timing studies have not extended to luminosities much below the hard state transition.

The hard state accretion geometry, the emission mechanisms, and the connection between the accreting material and the compact jet outflow that is present in the hard state for at least some BHCs (Fender 2001) are all currently active areas of debate. Several theoretical models have been suggested. One possibility is that a quasi- 
spherical, optically thin region forms in the inner portion of the accretion flow as in advection-dominated accretion flow (ADAF) models (Naravan, McClintock \& Yi 1996). The X-ray emission is predominantly due to thermal Comptonization in the hard state for ADAF models and for more generic "sphere+disk" models, and some success has been achieved in comparing such models to observations (Esin. McClintock \& Naravan 1997; Nowak et al. 1999). However, for a range of disk viscosities, the ADAF model is convectively unstable (Ouataert \& Gruzinov 2000), and this should be considered before concluding that any ADAF or sphere+disk model provides the correct physical description. Another possibility that implies a much different site for X-ray production and a different accretion geometry is that the X-ray emission is due to magnetic flares above the disk (Galeev. Rosner \& Vaiana 1979; di Matteo, Celotti \& Fabian 1999). Although the presence of a jet is not incorporated into the basic models described above, some recent work describes how a jet may arise from a "magnetic corona" (Merloni \& Fabian 2002). Other models have focused on the emission from the compact jet, and one suggestion is that the $\mathrm{X}$-ray emission is produced in the jet via a synchrotron mechanism (Markoff. Falcke \& Fender 2001). Improving the luminosity coverage for the hard state is important for providing tests of these models. In addition, it is currently unclear whether the same physical model can apply to these systems in the hard state and in quiescence. Major physical changes to the system could be seen as another state transition at very low luminosities. On the other hand, the lack of a sharp transition could indicate that quiescence is simply a low luminosity extension of the hard state as has been previously suggested for GX 339-4 (Corbel et al. 2000; Kong et al. 2000).

We chose to carry out our program by observing XTE J1650-500. This was a new BHC transient when it was discovered in outburst in 2001 (Remillard 2001). Although the compact object mass is not well-constrained so that it has not been confirmed that this is a black hole system, its X-ray properties strongly suggest that it harbors a black hole (Tomsick et al. 2003; Homan et al. 2003; Kalemci et al. 2003b). During its outburst, the source was optically identified (Castro-Tirado et al. 2001), and a radio counterpart was found (Groot et al. 2001). In quiescence, a radial velocity study was carried out, indicating a binary orbital period of $5.1 \mathrm{hr}$ and an optical mass function of $0.64 \pm$ $0.03 \mathrm{M}_{\odot}$ (Sanchez-Fernandez et al. 2002). The value for the mass function is relatively low for a black hole system and could indicate that the system contains a relatively low mass black hole, has a low binary inclination $\left(i<40^{\circ}\right)$, or both. We previously reported on RXTE observations of XTE J1650 500 , including a study of its timing properties near the softto-hard state transition (Kalemci et al. 2003b). We also reported on 14 day X-ray oscillations and $\sim 100 \mathrm{~s}$ X-ray flares that were detected with $R X T E$ near the end of the outburst (Tomsick et al. 2003). The current work focuses on our studies of the source with Chandra.

\section{OBSERVATIONS AND ANALYSIS}

We obtained 3 Chandra observations of XTE J1650-500 near the end of its 2001-2002 outburst, and the times of the observations are marked on the long-term X-ray light curve shown in Figure 1 We compiled the $3-20 \mathrm{keV}$ light curve in Figure 1 using all the observations of XTE J1650-500 that were made with the Proportional Counter Array (PCA) on

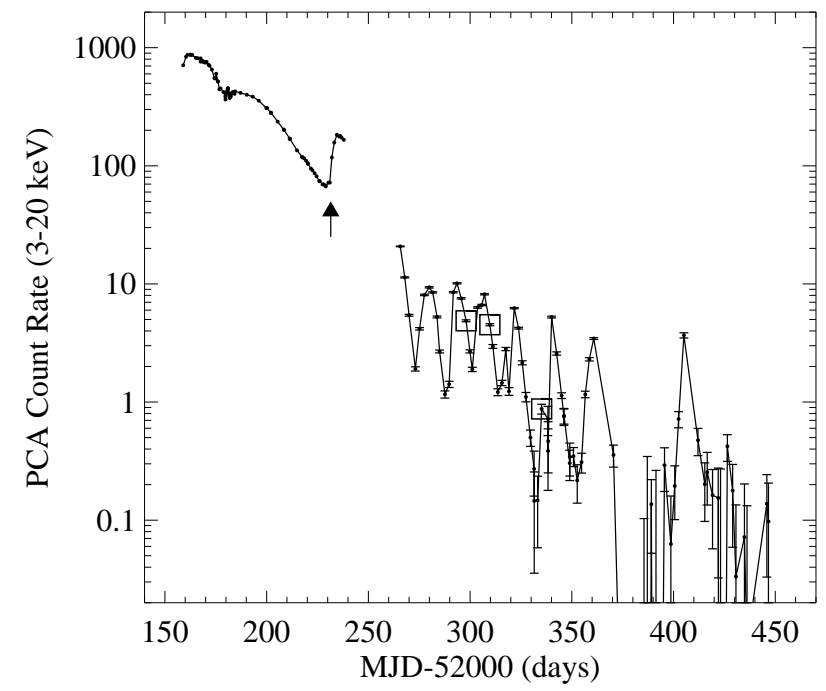

FIG. 1. - The 3-20 keV light curve for the 2001-2002 XTE J1650-500 outburst using PCA data from all the RXTE observations. The time of the soft-to-hard state transition, which occurred on MJD 52231.5, is marked with an arrow, and the gap in observations that is centered near MJD 52250 is due to solar angle constraints. After the gap, the source exhibited 14 day oscillations (Tomsick et al. 2003). The 3 Chandra observations occurred during these oscillations and are marked with squares.

RXTE. The 3 Chandra observations were made 67,78 , and 104 days after the soft-to-hard state transition (Homan et al. 2003; Kalemci et al. 2003b), during the time when the source was exhibiting large-amplitude flux oscillations with a characteristic time scale of 14 days (Tomsick et al. 2003). All 3 observations were made at flux levels well below the level of the soft-to-hard state transition.

The Chandra observations were made using the Advanced CCD Imaging Spectrometer (ACIS). In each case, the source was placed on one of the back-illuminated ACIS chips (S3). The first two observations (1 and 2), were made with the High Energy Transmission Grating (HETG) inserted, and each observation lasted approximately $10 \mathrm{ks}$ (see Table 1 for exact times and other detailed information about the observations). To increase the throughput, the grating was not used for observation 3, making photon pile-up (Davis 2001) a concern. To mitigate the effects of pile-up, we reduced the CCD exposure time from $0.4 \mathrm{~s}$ per frame from the nominal $3.2 \mathrm{~s}$ per frame by using a 1/8 CCD sub-array. In addition, the telescope was pointed $2^{\prime} .7$ away from the target, slightly blurring the point spread function (PSF) for XTE J1650-500. An exposure time of $18 \mathrm{ks}$ was obtained for observation 3 . In addition to the Chandra observations, shorter 1.8-2.4 ks RXTE observations were co-ordinated to cover part of each Chandra observation.

\subsection{Chandra Analysis}

For the two grating observations, we used the "level 2" event list produced by the standard data processing (ASCDS v6.5.1 using CALDB v2.11). For both observations, we applied a standard correction to the ACIS-S4 chip to correct a flaw in the chip readout. For this, as well as the data processing described below, we used the CIAO (Chandra Interactive Analysis of Observations) v2.3 software and CALDB (Calibration Data Base) v2.21. Prior to extracting the grating spectra, we produced background light curves. For observation 1 
only, we found a few background flares where the count rate increased by $50-100 \%$ for a time period of less than $50 \mathrm{~s}$. Due to their short duration, the flares make only a small contribution to the total background. Thus, we did not remove these times in the subsequent analysis, and the full integration time was used for both observations.

For observations 1 and 2, we produced $0.3-8 \mathrm{keV}$ zero order images. We used the source detection algorithm wavdetect to search for sources in a 2 by 2 arcminute region around the Chandra aim-point, and we only detect the target in each case. The observation 1 and 2 source positions are consistent with one another, and we obtain a mean source position of R.A. $=16 \mathrm{~h} 50 \mathrm{~m}$ 00s.98, Dec. $=-49^{\circ} 57^{\prime} 43^{\prime \prime} .6$ (equinox 2000.0) with an uncertainty of $0^{\prime \prime} .6$. This position is consistent with the previously reported radio (Groot et al. 2001) and optical (Castro-Tirado et al. 2001) positions, and we note that the Chandra position is somewhat more accurate than the $3^{\prime \prime}$ and $2^{\prime \prime}$ radio and optical positions, respectively. We conclude that the detected source is indeed XTE J1650-500. Given the recent detections of X-ray jets from XTE J1550 564 (Corbel et al. 2002) and 4U 1755-338 (Angelini \& White 2003), we examined the XTE J1650-500 images for X-ray extensions in the vicinity of the source, but we do not see any features that are likely to be jets. Using the zero order ACIS response matrix and a spectral shape similar to that observed for the XTE J1550-564 jet ( $\Gamma=1.6$ with interstellar absorption), the upper limit on the $0.3-8 \mathrm{keV}$ absorbed flux is $6 \times 10^{-14} \mathrm{erg} \mathrm{cm}^{-2} \mathrm{~s}^{-1}$ at positions $10^{\prime \prime}$ from XTE J1650-500.

We used the CIAO routine tgextract for the extraction of grating spectra and the CIAO routines mkgrmf and mkgarf to produce response matrices. Combining the +1 and -1 MEG (Medium Energy Grating) $0.7-7 \mathrm{keV}$ spectra yields 4786 counts in 10001 seconds for observation 1 and 4342 counts in 9509 seconds for observation 2. For the +1 and -1 HEG (High Energy Grating) 1-9 keV spectra, there are 2706 counts and 1872 counts for observations 1 and 2, respectively. We also produced estimated background spectra; however, we find that the background is not significant with estimates of $\sim 20$ counts for the MEG and $\sim 11$ counts for the HEG $(0.4 \%$ of the detected counts in both cases). The higher MEG and HEG orders contain between $\sim 30$ and $\sim 200$ counts (source plus background). For each observation, the zeroth order contains $\sim 2500$ counts, but there is a high level of photon pileup. The flux obtained from the grating spectra implies that the number of counts would be a factor of 3 higher without photon pile-up. Thus, due to the low number of counts in grating orders 2 and 3 and the high level of pile-up in the zeroth order, we focus on the order 1 spectra in this work. A final step in the extraction of the spectra is to apply a correction to the response matrix to account for the gradually changing low energy ACIS response. We used the script corrarf to apply this correction.

For observation 3, we used the "level 2" event list produced by the standard data processing (ASCDS v6.6.0 and CALDB v2.12). After inspecting an image including the full energy band, we produced a $0.3-8 \mathrm{keV}$ image to reduce the background level. With the 1/8 sub-array and only the S3 chip active, the field of view is approximately $1^{\prime}$ by $8^{\prime} .4$ (128 by 1024 pixels), and we used wavdetect to search for sources. We found 4 sources detected at greater than 4- $\sigma$ significance. The brightest source by far is at a position consistent with the XTE J1650-500 position found above for the grating observations. Although XTE J1650-500 appears to be elongated, we used CIAO to simulate an off-axis PSF, and we found that XTE
$\mathrm{J} 1650-500$ is consistent with a point-like source. The other 3 sources also appear to be point-like, and the closest source is more than $1^{\prime}$ from XTE J1650-500. Despite using an instrument setup designed to mitigate pile-up, the measured ACIS count rate of $0.493 \mathrm{~s}^{-1}$ for XTE J1650-500 (see Table 1) indicates that the data at the PSF core is moderately affected by photon pile-up. However, we were still able to use this data for spectral and timing analysis as described below and in Appendix A.

\subsection{RXTE Analysis}

We initially performed spectral and timing analysis for the 3 $R X T E$ observations that were made during the Chandra observations. Using the "Standard 2" PCA data, we extracted energy spectra using scripts developed at UC San Diego and the University of Tübingen that incorporate the standard software for RXTE data reduction (FTOOLS v5.2). We used the 2002 February release of the "Faint Source" model for background subtraction. Obtaining the best possible background subtraction is important for this work because of the low source count rates. To achieve this, we used Standard 2 data from the top anode layer only and excluded data from PCU 0 , which has a higher background level due to the loss of its propane layer ${ }^{4}$.

In addition to the 3 RXTE observations, we extracted energy spectra for the final 8 observations for which the PCA count rates are shown in Figure 11. For these observations, the mean 5-8 keV PCA count rate is $0.021 \pm 0.018 \mathrm{~s}^{-1}$ per Proportional Counter Unit (PCU) using all three anode layers, indicating that the rate is consistent with zero at slightly more than the 1- $\sigma$ level. We determined this rate in order to make a comparison to the rate expected from the Galactic ridge emission at the Galactic latitude of XTE J1650-500 $\left(b=-3.44^{\circ}\right)$. Valinia \& Marshall (1998) have determined the level of Galactic ridge emission (in PCA count rate) averaged over Galactic longitude as a function of $b$ (see Figure 2 of Valinia \& Marshall 1998). They found that the distribution could be described by narrow and broad gaussian components, and only the broad gaussian contributes at the Galactic latitude of XTE J1650-500. Using the parameters for the broad gaussian leads to a best estimate of the 5-8 keV Galactic ridge rate of $0.71 \mathrm{~s}^{-1}$ for all anode layers and 5 PCUs. Scaling the observed count rate given above $\left(0.021 \mathrm{~s}^{-1}\right)$ to 5 PCUs gives $0.11 \pm 0.09 \mathrm{~s}^{-1}$, which is significantly below the best estimate for the Galactic ridge emission. Thus, we conclude that the Galactic ridge emission can easily explain the low level of emission we detect for the final 8 observations. As this analysis shows that it is likely that XTE J1650-500 does not contribute to the flux detected during these observations, we produced an average spectrum (top anode layer only) for the final 8 observations and subtracted it from the PCA spectra for the 3 RXTE observations that were simultaneous with the Chandra observations. The effect to the continuum is small; however, the Galactic ridge emission contains a strong iron emission line at $6.7 \mathrm{keV}$, and this line is apparent in the average spectrum for the final 8 observations.

Finally, we analyzed the data from the 15 RXTE observations taken between MJD 52265 and MJD 52298, which is after the gap in $R X T E$ coverage and before the Chandra observations began (see Figure 1). We processed the PCA data as described above, including the subtraction of the Galactic ridge emission from the energy spectrum. For the spectral

\footnotetext{
4 See http://lheawww.gsfc.nasa.gov/users/craigm/pca-bkg/bkg-users.html for a detailed analysis of the background model performance.
} 


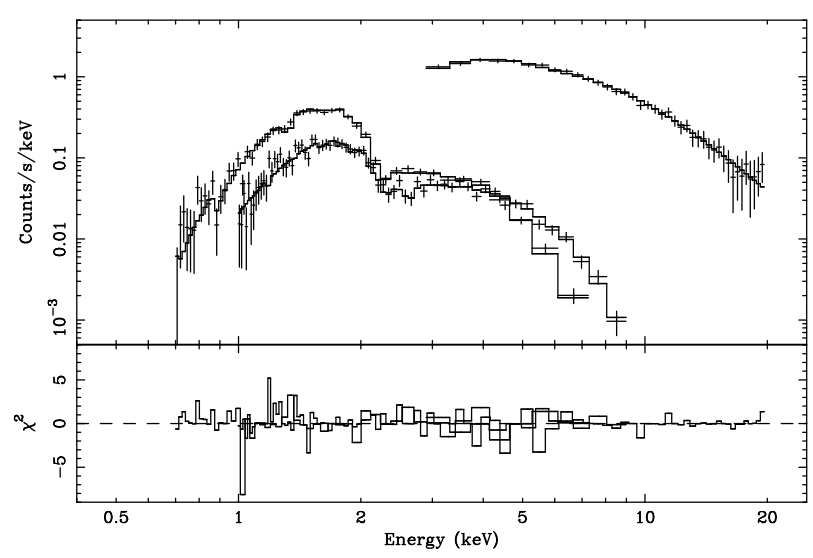

FIG. 2.- The observation 1 Chandra and PCA energy spectra fitted with an absorbed power-law model. The Chandra instruments used are the ACIS/HETG/MEG (0.7-7 keV) and the ACIS/HETG/HEG (1-9 keV). The measured power-law photon index is $\Gamma=1.66 \pm 0.05$. The bottom panel shows the residuals.

analysis described in $\S 3.3$, we also removed flares from 2 of the observations as described in Tomsick et al. (2003).

\section{RESULTS}

\subsection{Energy Spectra at Low Flux Levels}

Using XSPEC 11.2, we performed simultaneous leastsquares fits to the Chandra and PCA spectra for all three observations. For the PCA, we included $0.6 \%$ and $0.3 \%$ systematic errors for energy bins below and above $8 \mathrm{keV}$, respectively, as described in our previous work (Tomsick et al. 2003). We began by fitting the observation 1 spectrum with a power-law with interstellar absorption. We also included a constant factor to allow for the possibility that the overall normalization is different for the PCA and Chandra, but we assumed that the overall MEG and HEG normalizations agree with each other. As shown in Table 2 a power-law model with $\Gamma=1.66 \pm 0.05$ and $N_{\mathrm{H}}=(6.7 \pm 0.5) \times 10^{21} \mathrm{~cm}^{-2}$ (errors are $90 \%$ confidence, $\left.\Delta \chi^{2}=2.7\right)$ provides a good fit to the spectrum $\left(\chi^{2} / \nu=116 / 155\right)$. The other continuum models we tried do not provide acceptable fits. Thermal models, such as a blackbody or a disk-blackbody (Makishima et al. 1986), result in reduced $\chi^{2}$ values of 8.4 and 2.3, respectively. Figure 2 shows the observation 1 spectrum and residuals with the power-law fit. No clear features are seen in the residuals, and we note that the fit is not improved by adding a soft component, a high energy cutoff, or an iron emission line. The upper limit on the equivalent width of a narrow (where "narrow" is set by the binning shown in Figure 2) $\mathrm{Fe} \mathrm{K} \alpha$ emission line at $6.4 \mathrm{keV}$ is $161 \mathrm{eV}$.

Table 1 and Figure 1 indicate that the count rates are very similar for observations 1 and 2. We fitted the observation 2 spectrum, and Table 2 shows that the spectral parameters do not change significantly between the two observations. Also, in both cases, the spectral fits indicate that the PCA/Chandra normalization factor is near 1.12. As the PCA exposure only covers part of the Chandra observation, we checked to see if this difference could be due to source variability. We extracted a grating spectrum for observation 1 that is strictly simultaneous with the PCA exposure. During this time, the MEG count rate is $0.483 \pm 0.016 \mathrm{~s}^{-1}(0.7-7 \mathrm{keV})$, which is consistent with the rate for the full observation $\left(0.479 \pm 0.007 \mathrm{~s}^{-1}\right)$. Thus, we conclude that the overall normalizations are different for

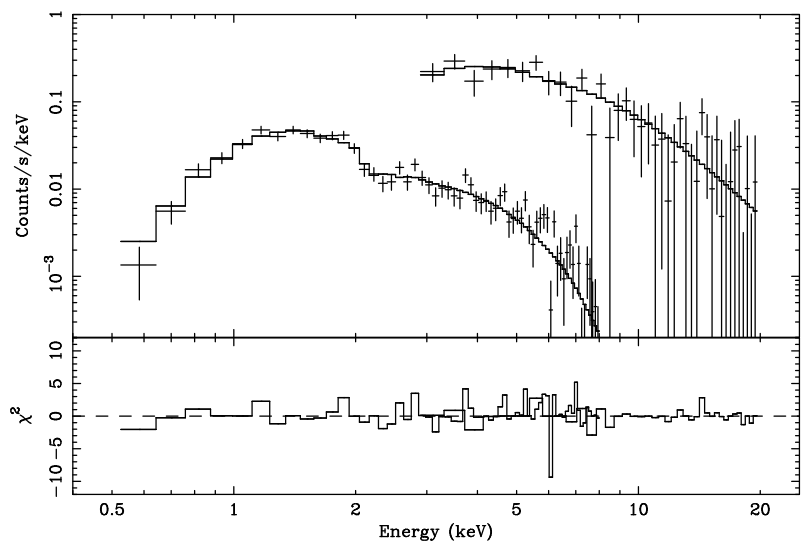

FIG. 3.- The observation 3 Chandra and PCA energy spectra fitted with an absorbed power-law model. We obtained the Chandra spectrum (0.5-8 $\mathrm{keV}$ ) with direct ACIS imaging. The measured power-law photon index is $\Gamma=1.93 \pm 0.13$. The bottom panel shows the residuals.

the PCA and the Chandra gratings, and we note that this is not surprising since it has been previously determined that the PCA normalization is high relative to previous instruments (Tomsick et al. 1999).

Due to the presence of photon pile-up in the PSF core, we extracted the observation 3 ACIS spectrum after removing the counts detected in the PSF core. We extracted the source counts from an elliptical annulus centered on the XTE J1650-500 position. We used an inner ellipse with a semimajor axis of $1^{\prime \prime} .3$ and a semi-minor axis of $0^{\prime \prime} .9$, and an outer ellipse with a semi-major axis of $4^{\prime \prime} .9$ and a semi-minor axis of $3^{\prime \prime} .1$. We rotated the ellipses to match the off-axis Chandra PSF. This region contains 1573 counts compared to the 9000 counts obtained when the core is included (see Table 1), and we are confident that the photons from the elliptical annulus do not suffer from pile-up. However, it is critical to realize that ACIS response matrices produced by the standard CIAO software do not account for the size or shape of the source extraction region or the fact that the Chandra PSF is energy dependent. We corrected the ACIS response matrix for this using the Chandra Ray Tracing software (ChaRT) as described in Appendix B. When producing the response matrix we also applied the low energy ACIS response correction for detector evolution as described above for the grating spectra.

We fitted the observation 3 ACIS+PCA spectrum with an absorbed power-law model as shown in Figure 3 This model provides a good fit to the data $\left(\chi^{2} / \nu=104 / 100\right)$ with parameter values of $N_{\mathrm{H}}=(6.4 \pm 0.7) \times 10^{21} \mathrm{~cm}^{-2}$ and $\Gamma=1.93 \pm 0.13$ (see Table 2). The measured column density is consistent with the value obtained from the grating observations. Thus, we re-fitted the spectrum after fixing the column density to the value found using the grating spectra $\left(N_{\mathrm{H}}=6.7 \times 10^{21} \mathrm{~cm}^{-2}\right)$, and we obtain $\Gamma=1.96 \pm 0.09$. Also, for consistency with observations 1 and 2, we used the PCA normalization for observation 3 divided by the PCA/grating normalization factor of 1.12 to determine the observation 3 flux reported in Table 2 The spectral results indicate that the XTE J1650-500 keeps its power-law shape but softens somewhat at low luminosity.

\subsection{Power Spectra at Low Flux Levels}

We examined the PCA and Chandra light curves for all three observations. As shown in Figure 4 clear variability is present in the PCA light curves for observations 1 and 2 and 

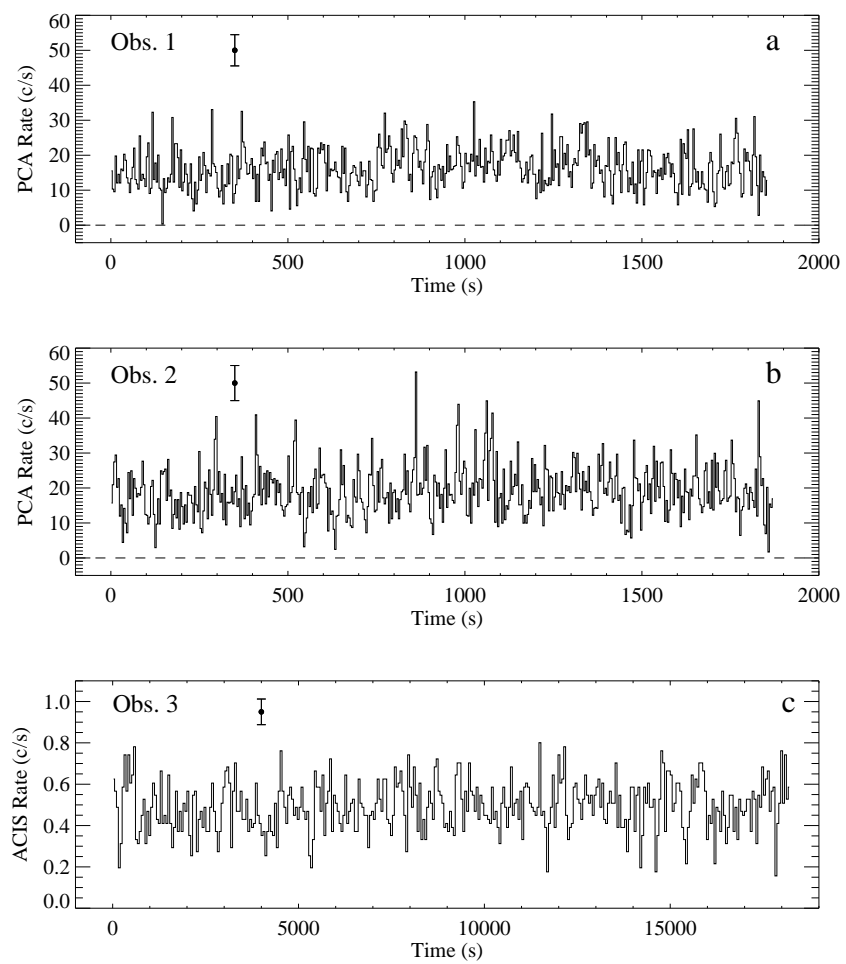

FIG. 4.- PCA (panels a and b) and ACIS (panel c) light curves for the 3 observations. The energy bands are $3-20 \mathrm{keV}$ and $0.5-8 \mathrm{keV}$ for the PCA and ACIS, respectively, and the light curves have been background subtracted. In each case, a representative error bar is shown.

in the ACIS light curve for observation 3. The plotted 3-20 $\mathrm{keV}$ PCA light curves (panels a and b) have $4 \mathrm{~s}$ time bins, and we note that the count rate is higher than reported in Table 1 because we used all anode layers and PCUs 0,2 , and 3 rather than just the top layer and PCUs 2 and 3. While the quality of the background estimate is slightly worse when PCU 0 and all layers are included, the higher count rate allows for tighter constraints on the timing properties. We also produced grating light curves (MEG+HEG) for observations 1 and 2. Although the statistical quality of the PCA light curves is superior to that of the grating light curves, we use the grating light curves for the analysis below as they allow us to study the energy dependence of the timing properties. Figure 4, shows the 0.5-8 $\mathrm{keV}$ ACIS light curve for observation 3 with $\sim 50$ s time bins. We also examined the PCA light curve for observation 3, but it is background dominated and is not useful for timing analysis.

For observations 1 and 2, we produced power spectra using PCA data taken in a high time resolution "event" mode (labeled E_125us_64M_0_1s). These data consist of an event list with photons time tagged to within $125 \mu \mathrm{s}$ and 64 energy bins. First, we used the data in the $3-20 \mathrm{keV}$ band to produce Leahy normalized power spectra (Leahy et al. 1983) for observations 1 and 2 separately. In each case, we averaged power spectra from four $460 \mathrm{~s}$ light curve segments and used a Nyquist frequency of $64 \mathrm{~Hz}$. No noise in excess of the Poisson level is present above $\sim 1 \mathrm{~Hz}$, and there were no clearly significant differences between the power spectra for the two observations. Thus, we produced another Leahy normalized power spectrum (again with a $3-20 \mathrm{keV}$

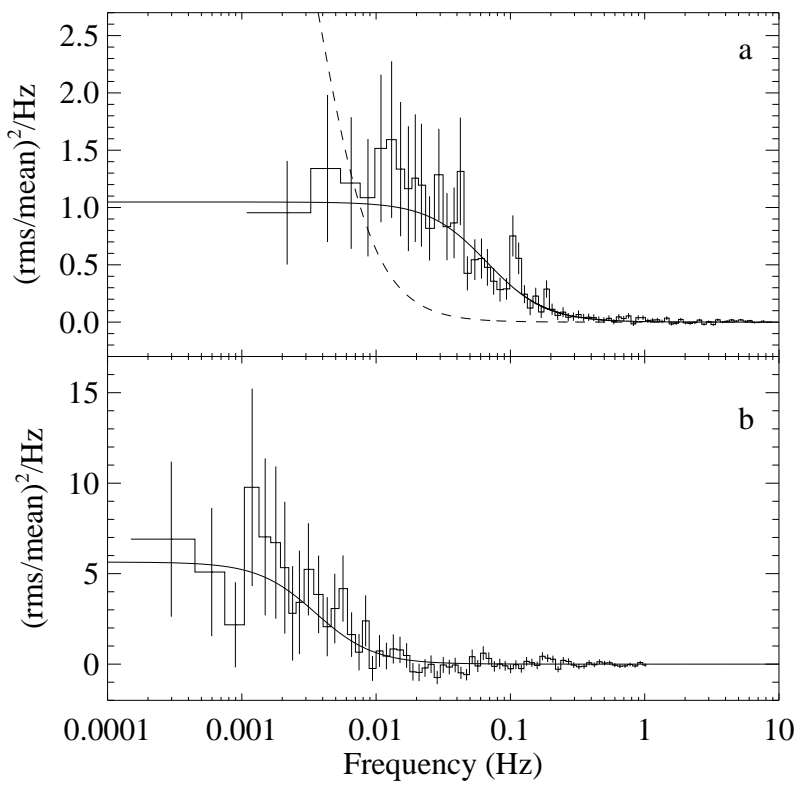

FIG. 5.- (a) PCA power spectrum for observations 1 and 2 combined. The solid line shows the best fit zero-centered Lorentzian, and the power spectrum shows marginal (between 2 and 3- $\sigma$ ) evidence for a QPO at $0.11 \mathrm{~Hz}$. (b) ACIS power spectrum for observation 3 with the best fit zero-centered Lorentzian. In panel a, the dashed line shows the model used for observation 3 to illustrate that the power shifts to lower frequency for observation 3 .

energy band) consisting of the eight $460 \mathrm{~s}$ light curve segments from observations 1 and 2 with a Nyquist frequency of $8 \mathrm{~Hz}$. We converted to the rms normalization (Mivamoto et al. 1994), and this power spectrum is shown in Figure 5 5 . The power spectrum is dominated by a band-limited noise component, and such a component has typically been fitted with a broken power-law or a zero-centered Lorentzian in previous work (Belloni. Psaltis \& van der Klis 2002). A zero-centered Lorentzian with a half-width (FWHM/2) of $0.067 \pm 0.007 \mathrm{~Hz}$, and a fractional rms amplitude of $33.1 \% \pm 1.1 \%$ (see Table 3 provides a reasonably good fit to the power spectrum $\left(\chi^{2} / \nu=93 / 72\right)$. We note that the half-width of a zerocentered Lorentzian is equivalent to the "peak" frequency that is sometimes quoted when fitting Lorentzians to power spectra (Belloni. Psaltis \& van der Klis 2002; Kalemci et al. 2003b). Positive residuals are present near $0.1 \mathrm{~Hz}$, possibly indicating the presence of a quasi-periodic oscillation (QPO). The addition of a second Lorentzian at $0.11 \mathrm{~Hz}$ improves the quality of the fit, resulting in $\chi^{2} / \nu=83 / 69$; however, this improvement corresponds to a detection at only the $96 \%$ confidence level. Although we repeated this analysis several times with different frequency binnings, we consistently found that the QPO is only detected at a level of between 2 and 3- $\sigma$. Thus, we take the measured rms amplitude of $12 \%$ as an upper limit on a possible QPO at $0.11 \mathrm{~Hz}$.

Using the grating light curves, we produced a $0.7-9 \mathrm{keV}$ power spectrum for observations 1 and 2 by averaging eight $2414 \mathrm{~s}$ light curve segments. The time resolution is set by the interval of time between chip read-outs, and this interval is $2.54104 \mathrm{~s}$ for these observations, giving a Nyquist frequency of $0.2 \mathrm{~Hz}$. Although the time resolution and the sta- 
tistical quality are lower for the grating light curves than for the PCA data, using the grating data allows us to extend the timing study to lower energies and frequencies. After converting to rms normalization, we fitted the grating power spectrum with a zero-centered Lorentzian, and the fit is acceptable $\left(\chi^{2} / \nu=25 / 23\right)$. As shown in Table 3 , the half-width is $0.079 \pm 0.020 \mathrm{~Hz}$, and the fractional $\mathrm{rms}$ is $30.1 \% \pm 2.5 \%$. Thus, the parameters for the $0.7-9 \mathrm{keV}$ power spectrum are consistent with those for the 3-20 keV PCA power spectrum.

We also produced a power spectrum using the observation 3 ACIS data. Using a $0.5-8 \mathrm{keV}$ light curve with a time resolution of $0.44104 \mathrm{~s}$, we produced a Leahy normalized power spectrum with a Nyquist frequency of $1.13 \mathrm{~Hz}$, consisting of the average of six $3350 \mathrm{~s}$ light curve intervals. In the Leahy normalization, Poisson noise leads to a power level of 2.0; however, at high frequencies $(0.1-1 \mathrm{~Hz})$ the ACIS power spectrum is consistent with a constant level of 1.77. We performed simulations described in Appendix A showing that pile-up at the level present for observation 3 leads to a drop in the Poisson noise level from 2.0 to 1.78 . This, along with a small drop in the Poisson noise level caused by deadtime during chip read-outs (see Appendix A) explains our measurement of 1.77. Thus, when converting the ACIS power spectrum from the Leahy normalization to the rms normalization, we subtracted a value of 1.77 rather than the usual value of 2.0. The rms normalized power spectrum fitted with a zero-centered Lorentzian is shown in Figure 5 5 , and this model provides a good fit to the data $\left(\chi^{2} / \nu=56 / 65\right)$. The Lorentzian halfwidth is $0.0035 \pm 0.0010 \mathrm{~Hz}$ (see Table 3), which is significantly lower than the value obtained for observations 1 and 2 . This indicates that, as the flux drops, the band-limited noise moves to lower frequencies. This fact is illustrated in Figure 5 a, where the model used to fit the observation 3 power spectrum is plotted as a dashed line on the power spectrum for observations 1 and 2 . The fit to the observation 3 power spectrum indicates a fractional rms amplitude of $17.7 \% \pm 1.7 \%$, but this (16\%) should be taken as a lower limit on the actual value since the noise level is reduced by pile-up. From our simulations, we estimate that the actual rms amplitude is near $19 \%$. We also produced a power spectrum using only the 1573 counts from the region of the CCD that is free of pile-up. Fitting the power spectrum with a zero-centered Lorentzian, the half-width is not well-constrained, but it is consistent with the previous fit, and the rms amplitude is $19 \% \pm 7 \%$, giving an upper limit of $26 \%$. Thus, considering the fits to the power spectra with and without pile-up, we conclude that the rms is $19 \%_{-3 \%}^{+7 \%}$.

\subsection{Summary of XTE J1650-500 Hard State X-Ray Observations}

For observations 1-3, two of our main results are that the source softens ( $\Gamma$ changes from $1.66 \pm 0.05$ to $1.93 \pm 0.13$ ) and the band-limited noise component moves to lower frequencies (the Lorentzian half-width, FWHM/2, changes from $0.067 \pm 0.007 \mathrm{~Hz}$ to $0.0035 \pm 0.0010 \mathrm{~Hz}$ ) as the source flux decays. Thus, where possible, we determined the values of these parameters ( $\Gamma$ and $\mathrm{FWHM} / 2$ ) for other $R X T E$ observations of XTE J1650-500 in the hard state during outburst decay. For the 7 observations made immediately after the soft-to-hard state transition on MJD 52231.5 (see Figure 1, we use the spectral parameters from Kalemci (2002). These energy spectra are dominated by a power-law component, but it should be noted that a soft component is also present. For these 7 observations, the timing parameters come from
Kalemci et al. (2003b). In contrast to our Chandra/RXTE observations at low flux levels, several Lorentzians are required to describe the power spectra for most of the observations immediately after the soft-to-hard state transition. The lowest frequency component in those power spectra are nearly zerocentered Lorentzians, and we assume that they correspond to the band-limited noise component that we see in the low flux power spectra.

For the 15 RXTE observations after the gap in RXTE coverage and before the Chandra observations began, the 3-20 keV energy spectra are all well-described by a single powerlaw component with the column density fixed to the value of $N_{\mathrm{H}}=6.7 \times 10^{21} \mathrm{~cm}^{-2}$ found for the grating observations. For these observations, the $3-20 \mathrm{keV}$ flux varies from $1.2 \times 10^{-11}$ to $2.9 \times 10^{-10} \mathrm{erg} \mathrm{cm}^{-2} \mathrm{~s}^{-1}$, and the values of $\Gamma$ range from $1.69 \pm 0.02$ to $\sim 2.0$. The data suggest a trend of softening as the flux drops consistent with that observed with Chandra, but the errors on $\Gamma$ are large for the low flux observations. We produced power spectra for all 15 observations; however, we could not obtain reliable fit parameters for about half of the observations due to poor statistics, short observations, or flaring (Tomsick et al. 2003). In 8 cases, we obtained useful power spectra, and these are dominated by a band-limited component with a fractional rms amplitude near 30\%. We fitted these power spectra with a zero-centered Lorentzian to determine the half-width for comparison to the other observations. Figure 6 shows the measured values of the Lorentzian half-width vs. $3-9 \mathrm{keV}$ flux, and Figure $6 \mathrm{p}$ shows the halfwidth vs. the measured values of $\Gamma$.

For the data points above a half-width of $0.5 \mathrm{~Hz}$, there is a tight correlation between $\Gamma$ and the half-width with a linear correlation coefficient of 0.96 . The two points near $0.25 \mathrm{~Hz}$ also appear to be consistent with this correlation, but the correlation coefficient drops slightly to 0.91 when these points are included. It is clear that the correlation does not extend to lower frequencies as $\Gamma$ reaches a minimum near 1.65-1.70. In fact, the data at frequencies between $0.03 \mathrm{~Hz}$ and $0.3 \mathrm{~Hz}$ suggest the possibility of an anti-correlation between $\Gamma$ and the half-width, but the linear correlation coefficient is only -0.41 . We checked to see if it is valid to compare the Chandra/RXTE point in this frequency range to the $R X T E$ only points by fitting the $R X T E$ spectra alone for observations 1 and 2. Fixing $N_{\mathrm{H}}$ to $6.7 \times 10^{21} \mathrm{~cm}^{-2}$, we obtained values of $\Gamma$ consistent with those found using Chandra and RXTE together. Although the anti-correlation is not statistically significant, the distribution of the values of $\Gamma$ for the $0.03-0.3 \mathrm{~Hz}$ points is also not well-described by a constant at their weighted average of $\Gamma=1.70 \pm 0.01$. Thus, we conclude that there is some scatter in $\Gamma$ for the $0.03-0.3 \mathrm{~Hz}$ points, but we cannot establish a definite relationship between $\Gamma$ and the Lorentzian half-width in this frequency regime.

\section{DISCUSSION}

One of the main motivations for this work is to measure the X-ray properties of an accreting black hole in the relatively unexplored luminosity range between the transition to the low-hard (or hard) state and the quiescent state. Such measurements allow us to address the question of whether these should be considered as two separate states or if the quiescent state is, in fact, a low luminosity version of the hard state. This question has important implications for whether there are significant physical changes in accreting black hole systems that occur at low luminosities such as changes in the geometry of the accretion flow and/or emission mechanisms. 

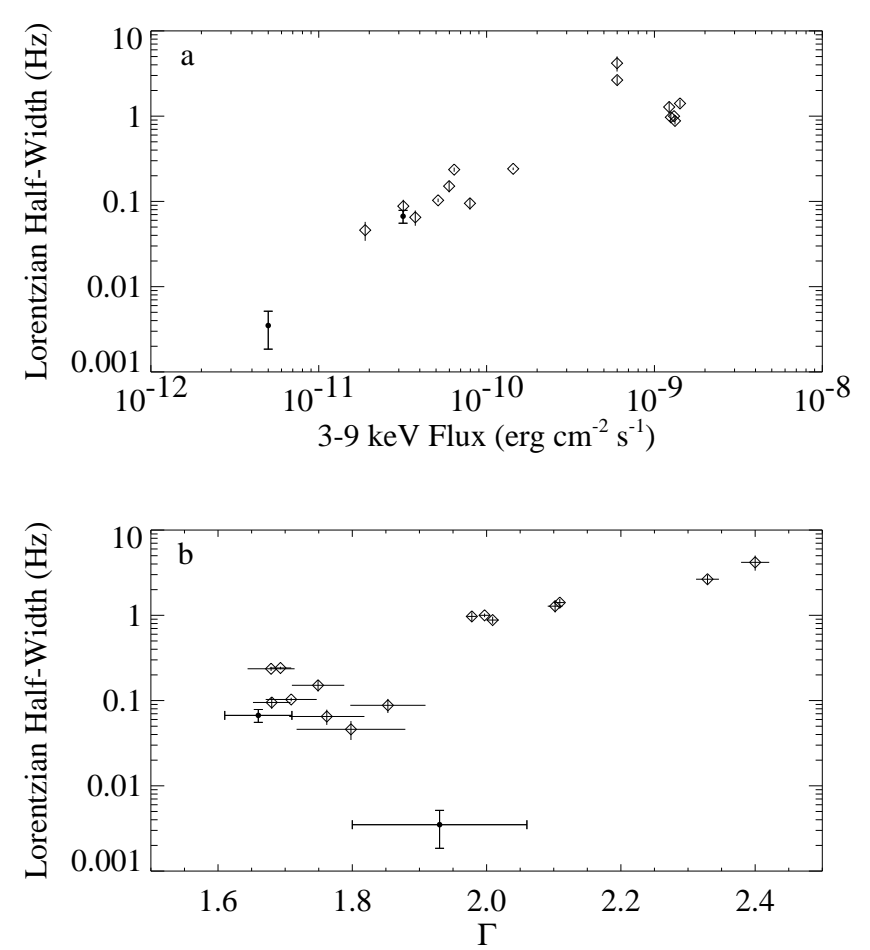

FIG. 6.- Measured parameters from Chandra and RXTE observations of XTE J1650-500 in the hard state. (a) The values of the Lorentzian half-width vs. the flux in the 3-9 keV energy band. (b) The half-width vs. the power-law photon index $(\Gamma)$. In both panels, the diamonds mark the measurements from observations with only RXTE data and the filled circles mark observations where we obtained both Chandra and RXTE data (one point for observations $1+2$ and one point for observation 3). The error bars on frequency and on $\Gamma$ are $90 \%$ confidence.

For XTE J1650-500, we have measured the spectral and timing properties of the source at 1-9 $\mathrm{keV}$ (absorbed) luminosities of $9 \times 10^{34} \mathrm{erg} \mathrm{s}^{-1}$ (observations 1 and 2) and $1.5 \times 10^{34} \mathrm{erg} \mathrm{s}^{-1}$ (observation 3 ). The quoted luminosities assume a fiducial source distance of $4 \mathrm{kpc}$, and we note that the unabsorbed luminosities are about $25 \%$ higher. As discussed in Tomsick et al. (2003), the likely distance for XTE J1650 500 is between $2 \mathrm{kpc}$ and $6 \mathrm{kpc}$. For the RXTE observation immediately after the soft-to-hard state transition, we used the spectral model described in Kalemci (2002) to extrapolate the PCA-measured flux into the 1-9 keV energy band, and we infer a transition luminosity of $7 \times 10^{36} \mathrm{erg} \mathrm{s}^{-1}$, which is inline with typical transition luminosities (Tanaka \& Shibazaki 1996). The observation 3 luminosity, which is a factor of 470 lower than the transition flux, is only about an order of magnitude above the quiescent luminosity of the black hole system that is brightest in quiescence (V404 Cyg, Garcia et al. 2001). Thus, our XTE J1650-500 observations cover a significant portion of the luminosity range between the soft-to-hard state transition and quiescence, but we note that the quiescent luminosity for XTE J1650-500 could be considerably lower than for V404 Cyg.

The trend observed for XTE J1650-500 that the bandlimited noise gradually shifts to lower frequency immediately after the transition to the hard state (Kalemci et al. 2003b) has also been observed for several other black hole systems (Kalemci et al. 2003a; Nowak, Wilms \& Dove 2002). The drop in frequency can be explained by accretion models con- sisting of an inner optically thin corona and an outer optically thick disk. As the mass accretion rate drops, the inner radius of the optically thick disk $\left(R_{\text {in }}\right)$ moves away from the black hole, causing a drop in the dynamical time scales and, thus, the characteristic frequencies for the system (di Matteo \& Psaltis 1999). After the XTE J1650-500 transition to the hard state, the Lorentzian half-width is correlated with the value of the power-law index down to at least a halfwidth of $1 \mathrm{~Hz}$ and possibly down to $0.25 \mathrm{~Hz}$ (see §3.3). Correlations between frequency (either half-width or QPO frequency) and $\Gamma$ have been seen before during RXTE observations of Cyg X-1, GX 339-4, and other black hole systems in their hard states (Gilfanov. Churazov \& Revnivtsev 1999; Revnivtsev, Gilfanov \& Churazov 2001; Kalemci 2002). As these authors argue, this correlation is expected for the disk and inner corona picture because the soft photons from the optically thick disk cool the corona, leading to a softer powerlaw index. Thus, as $R_{\text {in }}$ increases, and the characteristic frequencies drop, the corona is subject to a lower flux of soft photons from the disk, and the coronal spectrum hardens. This effect is predicted in both ADAF (Esin. McClintock \& Naravan 1997) models and in general disk and inner corona models such as the sphere+disk model (Nowak. Wilms \& Dove 2002) where the X-ray emission from the corona is predominantly due to thermal Comptonization.

While the half-width and $\Gamma$ are correlated above $0.25-1 \mathrm{~Hz}$, $\Gamma$ does not get any harder than 1.65-1.70 for XTE J1650-500, and the spectrum softens to $1.93 \pm 0.13$ as the half-width and flux continue to drop. This behavior can be interpreted within the ADAF or sphere+disk (henceforth ADAF/SD) models. The decrease in the half-width frequency indicates that the disk inner radius continues to recede; however, the disk flux is at such a low level that it no longer significantly cools the corona, and the spectrum does not continue to get harder. Instead, the hardness of the spectrum depends on the "Compton y-parameter," which is a function of the optical depth and the temperature of the corona (Rvbicki \& Lightman 1979). As the mass accretion rate drops, the coronal optical depth decreases, and a softer spectrum is predicted. Softer spectra have been observed at low luminosities for several black hole sources besides XTE J1650-500. XTE J1118+480 shows significant softening that starts at an X-ray luminosity of $2 \times 10^{35}$ $\operatorname{erg~s}^{-1}(d / 1.8 \mathrm{kpc})^{2}$, and, at the end of its 2001 outburst, XTE J1550-564 begins gradual softening at a luminosity of $2 \times 10^{36} \mathrm{erg} \mathrm{s}^{-1}(d / 5.3 \mathrm{kpc})^{2}$ (Kalemci 2002). Other examples of spectral softening at low luminosities include GS 1124-68 (Ebisawa et al. 1994), as well as some black hole sources in or close to quiescence (McClintock et al. 2003; Corbel et al. 2003; Kong et al. 2002; Tomsick, Corbel \& Kaaret 2001).

Although spectral fits with the ADAF model imply relatively large changes in $R_{\text {in }}$ between the soft and hard states, some previous black hole timing studies conclude that such changes, which can be as large as factors of 30-3000 (Esin. McClintock \& Naravan 1997), do not occur. For several black hole systems, di Matteo \& Psaltis (1999) find that the characteristic frequencies do not vary by more than a factor of 50 between states. For Keplerian orbits, $R_{\text {in }} \propto \nu^{-2 / 3}$, where $\nu$ is the characteristic frequency, so that a factor of 50 in frequency implies a change in $R_{\text {in }}$ by a factor of 14 , which is significantly less than indicated by ADAF fits. However, for XTE J1650-500, we find that the Lorentzian half-width changes by a factor of nearly 1200 while the source remains in the hard state, implying that $R_{i n}$ can change by as much as a 
factor of 113. Results of fast optical photometry for quiescent black hole systems suggest that $R_{\text {in }}$ may change by an even larger factor. For A 0620-00, Hynes et al. (2003) find that the optical power spectrum is dominated by band limited noise with a break frequency of $9.5 \times 10^{-4} \mathrm{~Hz}$, which is a factor of 3.7 lower than the lowest frequency we find for XTE J1650500 . If the frequency is, in fact, related to $R_{i n}$, then our result for XTE J1650-500 and the results of Hynes et al. (2003) imply that very large values of $R_{\text {in }}$ occur at low luminosities, and that the $R_{\text {in }}$ evolution occurs gradually and continues to change all the way down to quiescent luminosities. These results also imply that an extremely large value of $R_{\text {in }}$ is not required for a source to show hard state properties.

While the ADAF/SD models described above can explain many of the X-ray properties we see for XTE J1650-500, radio observations of XTE J1650-500 made after the transition to the hard state indicate the likely presence of a compact jet (Corbel et al., in prep.), which is not accounted for in these models. However, the "magnetic corona" model of Merloni \& Fabian (2002) also explains several of the XTE J1650-500 X-ray properties while predicting that energy stored in the corona should be available to drive strong outflows. In this model, the X-ray emission mechanism is also Comptonization, so that a high flux of disk photons leads to a softer energy spectrum. Thus, Merloni \& Fabian (2002) predict that the spectrum will harden as the mass accretion rate drops, and they also predict that the spectrum will soften at very low mass accretion rates as more of the coronal energy is carried away by the outflow. Recent work by Fender, Gallo \& Jonker (2003) suggests that we are observing XTE J1650-500 in a luminosity regime where this could occur. While the XTE J1650-500 spectral evolution is consistent with the Merloni \& Fabian (2002) model, and we also previously argued that this model may be able to account for the X-ray flares reported in Tomsick et al. (2003), it is unclear if the magnetic corona model can account for the fact that the observed characteristic frequency for XTE J1650500 changes by a factor of 1200 in the hard state. Perhaps this could indicate large variations in the size of the magnetic corona or in the sizes of the active coronal regions, but since $R_{i n}$ is not expected to change by a large factor in this model, we do not see an obvious explanation for the large frequency range.

A jet-dominated model has also been suggested for black holes in their hard state. In this model, the compact jet contributes a large fraction of the emission from the radio band up to X-ray energies, and a synchrotron emission mechanism is invoked (Markoff, Falcke \& Fender 2001). Evidence in favor of this model includes observations of correlated radio and X-ray flux for several black hole systems (Corbel et al. 2003: Gallo. Fender \& Poolev 2003), and good agreement between the predictions of the model and the broadband GX 339-4 energy spectra over a large range of mass accretion rates (Markoff et al. 2003). In the model, X-rays are produced when electrons are accelerated at a shock that is $z_{s h} \sim 10^{3} R_{g}$ from the black hole, where $R_{g}=G M / c^{2}$ and $M$ is the black hole mass. The X-ray spectrum is predicted to have a powerlaw shape out to a cutoff energy, and the power-law index is set by the index of the electron energy distribution, $p$, where $d N / d E \propto E^{-p}$. Markoff et al. (2003) find that they can explain the radio/X-ray flux correlation for GX 339-4 by varying only the jet power, keeping $p$ and $z_{\text {sh }}$ constant. The fact that $\Gamma$ changes for XTE J1650-500 and also for GX 339-4
(Corbel et al. 2003), indicates that if this model is correct, $p$ likely does change with source luminosity, but we suspect that changes in $\Gamma$ are probably not a major problem for this model as one might expect $p$ to vary somewhat with jet power. On the other hand, one would expect that the characteristic frequencies of the system would be related to the size of the source. Assuming that the size of the X-ray emitting region is related to the location of the shock, $z_{s h}$, it may be difficult for the jet-dominated model to explain the large range of characteristic frequencies we observe for XTE J1650-500 without varying $z_{s h}$.

\section{CONCLUSIONS}

We have compared the emission properties of XTE J1650500 in the hard state over a large range of luminosities to ADAF/SD, magnetic corona, and jet-dominated models. Although the ADAF/SD model provides natural explanations for the X-ray spectral and timing properties, these models do not include outflows and thus cannot provide a complete description of the system. In addition, ADAFs may be convectively unstable as mentioned above. The magnetic corona model, which does incorporate a jet, predicts a similar spectral evolution to the ADAF/SD model, but it it unclear if it can explain the large change in the Lorentzian half-width that we see for XTE J1650-500. The jet-dominated model can explain X-ray/radio correlations that are seen for several black hole sources without varying $z_{s h}$; however, it is doubtful that the model can reproduce the observed X-ray timing properties with a constant value of $z_{s h}$.

Regardless of which model is correct, the fact that the general shape of the energy and power spectra remain the same while the parameters evolve indicates that the X-ray emitting region of the system likely keeps the same overall structure even though the parameter changes indicate that some physical properties change, such as the inner radius of the accretion disk, the size of the corona, or the shock location, considering the models described above. It is clear that the source retains its hard state $\mathrm{X}$-ray properties down to a luminosity of $1.5 \times 10^{34} \mathrm{erg} \mathrm{s}^{-1}$, and we do not see any evidence for a sharp transition to the quiescent state. Furthermore, our results for XTE J1650-500 combined with the determination that the optical power spectrum for A 0620-00 in quiescence exhibits band limited noise with an even lower characteristic frequency (Hynes et al. 2003) suggests that black hole transients keep the same overall structure into quiescence. Thus, these results favor the hypothesis that the quiescent state is simply a low luminosity version of the hard state.

JAT acknowledges useful discussions with M. A. Nowak, J. Wilms, and W. A. Heindl. We thank M. Garcia and H. Tananbaum for assistance in scheduling the Target of Opportunity observations with Chandra. We would like to thank all scientists who contributed to the Tübingen Timing Tools. JAT acknowledges partial support from NASA grant NAG513055 and Chandra award number GO2-3056X issued by the Chandra X-ray Observatory Center, which is operated by the Smithsonian Astrophysical Observatory for and on behalf of NASA under contract NAS8-39073. EK acknowledges partial support of TÜBTंAK. PK acknowledges partial support from NASA grant NAG5-7405. 


\section{REFERENCES}

Angelini, L., \& White, N. E., 2003, ApJ, 586, L71

Belloni, T., Psaltis, D., \& van der Klis, M., 2002, ApJ, 572, 392

Bradt, H. V., Rothschild, R. E., \& Swank, J. H., 1993, A\&AS, 97, 355

Castro-Tirado, A. J., Kilmartin, P., Gilmore, A., Petterson, O., Bond, I., Yock, P., \& Sanchez-Fernandez, C., 2001, IAU Circular, 7707

Corbel, S., Fender, R. P., Tzioumis, A. K., Nowak, M., McIntyre, V., Durouchoux, P., \& Sood, R., 2000, A\&A, 359, 251

Corbel, S., Fender, R. P., Tzioumis, A. K., Tomsick, J. A., Orosz, J. A., Miller, J. M., Wijnands, R., \& Kaaret, P., 2002, Science, 298, 196

Corbel, S., Nowak, M. A., Fender, R. P., Tzioumis, A. K., \& Markoff, S., 2003, A\&A, 400, 1007

Davis, J. E., 2001, ApJ, 562, 575

di Matteo, T., Celotti, A., \& Fabian, A. C., 1999, MNRAS, 304, 809

di Matteo, T., \& Psaltis, D., 1999, ApJ, 526, L101

Ebisawa, K., et al., 1994, PASJ, 46, 375

Esin, A. A., McClintock, J. E., \& Narayan, R., 1997, ApJ, 489, 865

Fender, R., Gallo, E., \& Jonker, P., 2003, Accepted by MNRAS, astro-ph/0306614

Fender, R. P., 2001, MNRAS, 322, 31

Galeev, A. A., Rosner, R., \& Vaiana, G. S., 1979, ApJ, 229, 318

Gallo, E., Fender, R. P., \& Pooley, G. G., 2003, MNRAS, 344, 60

Garcia, M. R., McClintock, J. E., Narayan, R., Callanan, P., Barret, D., \& Murray, S. S., 2001, ApJ, 553, L47

Gilfanov, M., Churazov, E., \& Revnivtsev, M., 1999, A\&A, 352, 182

Groot, P., Tingay, S., Udalski, A., \& Miller, J., 2001, IAU Circular, 7708

Homan, J., Klein-Wolt, M., Rossi, S., Miller, J. M., Wijnands, R., Belloni, T., van der Klis, M., \& Lewin, W. H. G., 2003, ApJ, 586, 1262

Hynes, R. I., Charles, P. A., Casares, J., Haswell, C. A., Zurita, C., \& Shahbaz, T., 2003, MNRAS, 340, 447

Kalemci, E., 2002, Ph.D. Thesis, Temporal Studies of Black Hole X-Ray Transients During Outburst Decay, UC San Diego

Kalemci, E., et al., 2003a, Submitted to ApJ

Kalemci, E., Tomsick, J. A., Rothschild, R. E., Pottschmidt, K., Corbel, S., Wijnands, R., Miller, J. M., \& Kaaret, P., 2003b, ApJ, 586, 419

Kong, A. K. H., Kuulkers, E., Charles, P. A., \& Homer, L., 2000, MNRAS, 312, L49

Kong, A. K. H., McClintock, J. E., Garcia, M. R., Murray, S. S., \& Barret, D., 2002, ApJ, 570, 277
Leahy, D. A., Darbro, W., Elsner, R. F., Weisskopf, M. C., Kahn, S., Sutherland, P. G., \& Grindlay, J. E., 1983, ApJ, 266, 160

Makishima, K., Maejima, Y., Mitsuda, K., Bradt, H. V., Remillard, R. A. Tuohy, I. R., Hoshi, R., \& Nakagawa, M., 1986, ApJ, 308, 635

Markoff, S., Falcke, H., \& Fender, R., 2001, A\&A, 372, L25

Markoff, S., Nowak, M., Corbel, S., Fender, R., \& Falcke, H., 2003, A\&A, 397,645

McClintock, J., \& Remillard, R., 2003, Review Article, astro-ph/0306213

McClintock, J. E., Narayan, R., Garcia, M. R., Orosz, J. A., Remillard, R. A., \& Murray, S. S., 2003, ApJ, 593, 435

Merloni, A., \& Fabian, A. C., 2002, MNRAS, 332, 165

Miyamoto, S., Kitamoto, S., Iga, S., Hayashida, K., \& Terada, K., 1994, ApJ, 435, 398

Narayan, R., McClintock, J. E., \& Yi, I., 1996, ApJ, 457, 821

Nowak, M. A., Vaughan, B. A., Wilms, J., Dove, J. B., \& Begelman, M. C., 1999, ApJ, 510, 874

Nowak, M. A., Wilms, J., \& Dove, J. B., 2002, MNRAS, 332, 856

Quataert, E., \& Gruzinov, A., 2000, ApJ, 539, 809

Remillard, R., 2001, IAU Circular, 7707

Revnivtsev, M., Gilfanov, M., \& Churazov, E., 2001, A\&A, 380, 520

Rybicki, G. B., \& Lightman, A. P., 1979, Radiative Processes in Astrophysics, New York, Wiley-Interscience, 1979. 393 p.)

Sanchez-Fernandez, C., Zurita, C., Casares, J., Castro-Tirado, A. J., Bond, I., Brandt, S., \& Lund, N., 2002, IAU Circular, 7989

Tanaka, Y., \& Shibazaki, N., 1996, ARA\&A, 34, 607

Tomsick, J., Corbel, S., Kalemci, E., \& Kaaret, P., 2003, ApJ, 592, 1100

Tomsick, J. A., Corbel, S., \& Kaaret, P., 2001, ApJ, 563, 229

Tomsick, J. A., \& Kaaret, P., 2000, ApJ, 537, 448

Tomsick, J. A., Kaaret, P., Kroeger, R. A., \& Remillard, R. A., 1999, ApJ, 512,892

Valinia, A., \& Marshall, F. E., 1998, ApJ, 505, 134

Weisskopf, M. C., Brinkman, B., Canizares, C., Garmire, G., Murray, S., \& Van Speybroeck, L. P., 2002, PASP, 114, 1

\section{APPENDIX A}

We performed simulations to study the effects of pile-up on the observation 3 ACIS power spectrum. The simulations have 3 main goals: 1 . To understand why the Poisson noise level for the actual Leahy normalized ACIS power spectrum is $1.77 ; 2$. To determine how much the fractional rms amplitude is reduced by pile-up; and 3. To check that pile-up does not alter the overall shape of the power spectrum. Our goals were accomplished via 3 simulations.

Simulation \#1: We produced a simulated Poisson noise light curve with the same time resolution as the actual data (but with much longer duration to improve the statistics). According to the binomial probabilities, we produced a piled-up light curve. The 2 adjustable parameters are the input count rate (before pile-up) and the probability that any pair of events occurring in the same time bin will pile-up. We adjusted these parameters to match the actual ACIS observation as shown in Figure 7 We produced a Leahy normalized power spectrum using the simulated light curve, and the measured Poisson level is 1.78.

Simulation \#2: In addition to the deadtime caused by pile-up, there is a fixed, instrumental deadtime that is independent of the event rate and is set by the CCD readout time of 41.04 milliseconds. To determine the effect of the readout time, we simulated a light curve where there is a probability (equal to the readout time divided by the $0.4 \mathrm{~s}$ exposure time per frame) that a photon that hits the detector will not be counted. We produced a Leahy normalized power spectrum using the simulated light curve, and the Poisson level is 1.97 .

Simulation \#3: Here, we made simulated light curves similar to those used for simulation \#1 except that we included white noise in excess of the Poisson noise. We produced a light curve free of pile-up and also a light curve with pile-up and made rms normalized power spectra for each. The fractional rms amplitude changes from $19 \%$ to $18 \%$ when pile-up effects are included. The shapes of the power spectra with and without pile-up are not significantly different.

In summary, these simulations demonstrate that the low Poisson noise level measured for the actual data is mainly due to pile-up effects, and there is also a small drop due to the CCD readout time. For noise levels similar to those we measure for observation $3(\sim 18 \%)$, pile-up only causes the fractional rms amplitude to drop by about $1 \%$, and the pile-up does not change the shape of the power spectrum.

\section{APPENDIX B}

Here, we describe how we corrected the observation 3 ACIS response matrix for the energy dependence of the Chandra PSF. We generated this correction using the web-based Chandra calibration tool "ChaRT" (Chandra Ray Tracing). For 13 different 


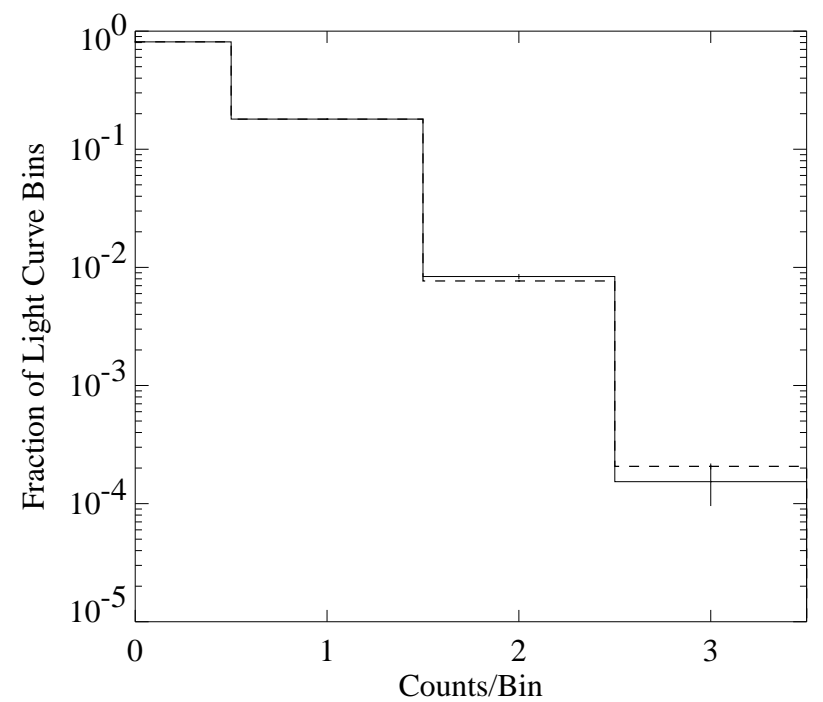

FIG. 7.- The solid histogram shows the fraction of $0.4 \mathrm{~s}$ observation 3 light curve bins with with $0,1,2$, or 3 counts/bin. The dashed histogram shows the same for a simulated light curve that includes the effects of photon pile-up. This illustrates that our simulated light curves are a good match to the actual data.

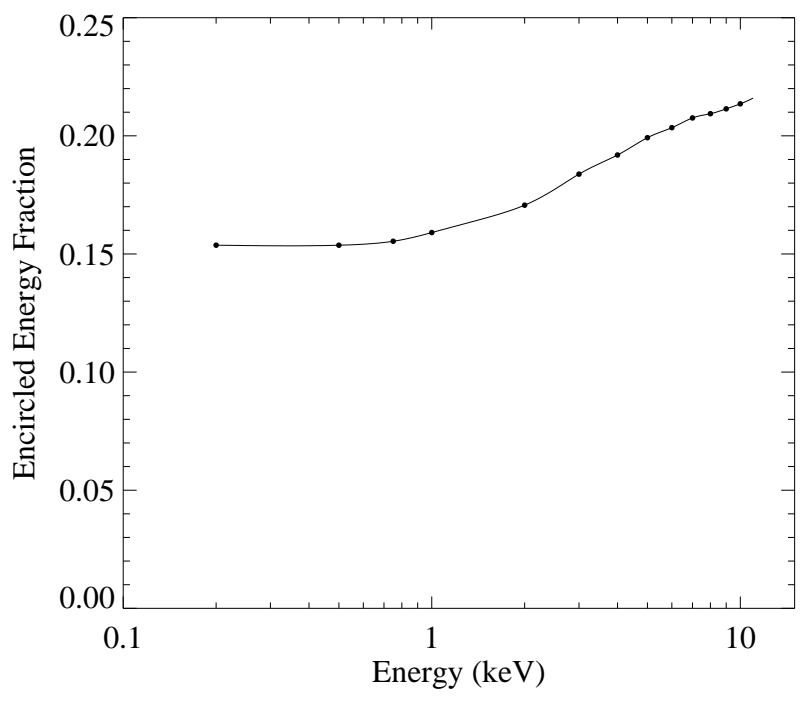

FIG. 8. - ChaRT (Chandra Ray Tracing) results for a source $2^{\prime} .7$ off-axis showing the fraction of counts in the elliptical annulus we used to extract the observation 3 ACIS spectrum (see text for the precise region parameters) as a function of energy. The points come from directly from the ChaRT simulations, and the solid line is the spline interpolation that was applied to the ACIS response matrix for observation 3 to account for the energy dependence of the Chandra PSF.

energies from 0.2 to $10 \mathrm{keV}$, we used ChaRT to simulate a monochromatic source at the position of XTE J1650-500, 2'.7 offaxis. For each energy, we determined the fraction of counts in the same elliptical annulus that we used to extract the XTE $\mathrm{J} 1650-500$ spectrum (see text), and the fractions are shown in Figure 8 We then used a spline interpolation to determine the fraction for each energy bin in the response matrix, and we applied the interpolated values to the response matrix. While this correction is necessary, we note that, in this case, it only changes the measurement of the power-law index from $\Gamma=1.79 \pm 0.13$ to $\Gamma=1.93 \pm 0.13$. Although we do not have a method of accurately determining the systematic error on this correction, even if the correction is only good to $10-20 \%$, the systematic error is much smaller than the statistical error. 
TABLE 1

Chandra AND RXTE OBSERVATIONS

\begin{tabular}{c|c|c|c|c|c}
\hline \hline $\begin{array}{c}\text { Obs./ } \\
\text { UT Date }\end{array}$ & MJD Start $^{a}$ & Instrument & $\begin{array}{c}\text { Energy Band } \\
(\mathrm{keV})\end{array}$ & $\begin{array}{c}\text { Exposure } \\
(\mathrm{s})\end{array}$ & $\begin{array}{c}\text { Count } \\
\text { Rate }^{b}\end{array}$ \\
\hline 1 & 52298.00688 & ACIS/HETG/MEG & $0.7-7$ & 10001 & $0.479 \pm 0.007$ \\
(2002 Jan. 23-24) & 52298.00688 & ACIS/HETG/HEG & $1-9$ & 10001 & $0.271 \pm 0.005$ \\
& 52298.03298 & RXTE/PCA & $3-20$ & 1904 & $9.42 \pm 0.14$ \\
\hline 2 & 52309.62048 & ACIS/HETG/MEG & $0.7-7$ & 9509 & $0.457 \pm 0.007$ \\
$(2002$ Feb. 4) & 52309.62048 & ACIS/HETG/HEG & $1-9$ & 9509 & $0.248 \pm 0.005$ \\
& 52309.71204 & RXTE/PCA & $3-20$ & 1872 & $8.69 \pm 0.14$ \\
\hline 3 & 52335.10444 & ACIS & $0.5-8$ & 18266 & $0.493 \pm 0.005^{d}$ \\
(2002 Mar. 2) & 52335.15312 & RXTE/PCA & $3-20$ & 2384 & $1.38 \pm 0.11$ \\
\hline
\end{tabular}

${ }^{a}$ Modified Julian Date (JD-2400000.5) at exposure midpoint.

${ }^{b}$ The MEG and HEG rates quoted are for the +1 and -1 grating orders combined.

${ }^{c}$ For this observation, we used a $1 / 8$ subarray and an offset pointing of $2^{\prime} .7$ to mitigate the effects of photon pile-up.

${ }^{d}$ This is the count rate for the source, including the core, which is moderately affected by photon pile-up. We estimate that the count rate would be $\sim 15 \%$ higher without pile-up.

TABLE 2

PARAMETERS FROM Chandra AND PCA SPECTRAL FITS

\begin{tabular}{c|c|c|c|c|c}
\hline \hline Obs. $^{a}$ & $\begin{array}{c}N_{\mathrm{H}} \\
\left(10^{21} \mathrm{~cm}^{-2}\right)\end{array}$ & $\Gamma$ & $\begin{array}{c}\text { Flux } \\
(1-9 \mathrm{keV})^{b}\end{array}$ & $\chi^{2} / \nu$ & $\begin{array}{c}\text { Fe K } \alpha \text { Line EW } \\
\text { Upper Limit }(\mathrm{eV})^{c}\end{array}$ \\
\hline 1 & $6.7 \pm 0.5$ & $1.66 \pm 0.05$ & $4.76 \times 10^{-11}$ & $116 / 155$ & $<162$ \\
2 & $6.7 \pm 0.5$ & $1.66 \pm 0.05$ & $4.46 \times 10^{-11}$ & $164 / 155$ & $<169$ \\
3 & $6.4 \pm 0.7$ & $1.93 \pm 0.13$ & $7.9 \times 10^{-12}$ & $104 / 100$ & $<212$ \\
3 & $6.7^{d}$ & $1.96 \pm 0.09$ & $8.0 \times 10^{-12}$ & $104 / 101$ & $<212$ \\
\hline
\end{tabular}

${ }^{a}$ These are parameters from absorbed power-law fits to the spectra. All errors and upper limits are $90 \%$ confidence $\left(\Delta \chi^{2}=2.7\right)$.

${ }^{b}$ The 1-9 $\mathrm{keV}$ absorbed flux in units of erg $\mathrm{cm}^{-2} \mathrm{~s}^{-1}$.

$90 \%$ confidence upper limit on the equivalent width of a narrow emission line at $6.4 \mathrm{keV}$.

${ }^{d}$ Fixed.

TABLE 3

PARAMATERS FOR THE POWER SPECTRA

\begin{tabular}{c|c|c|c|c|c}
\hline \hline Obs. $^{a}$ & Instrument & Energy Band (keV) & FWHM $/ 2^{b}$ & Fractional RMS (\%) & $\chi^{2} / \nu$ \\
\hline $1+2^{c}$ & PCA & $3-20$ & $0.067 \pm 0.007$ & $33.1 \pm 1.1$ & $93 / 72$ \\
$1+2$ & MEG+HEG & $0.7-9$ & $0.079 \pm 0.020$ & $30.1 \pm 2.5$ & $25 / 23$ \\
3 & ACIS & $0.5-8$ & $0.0035 \pm 0.0010$ & $19_{-3}^{+7}$ & $56 / 65$ \\
\hline
\end{tabular}

${ }^{a}$ These are parameters from fits with a zero-centered Lorentzian. Errors are $68 \%$ confidence.

${ }^{b}$ The half-width of the zero-centered Lorentzian in Hz.

${ }^{c}$ A QPO may be present near $0.11 \mathrm{~Hz}$ with a fractional rms of about $12 \%$. The significance of the QPO is only required at a confidence level of between $2-\sigma$ and $3-\sigma$. 